\title{
Quadratic Core-Selecting Payment Rules for Combinatorial Auctions
}

\author{
Robert W. Day \\ Operations and Information Management, School of Business, University of Connecticut, \\ Storrs, Connecticut 06269, bob.day@ business.uconn.edu \\ Peter Cramton \\ Economics Department, University of Maryland, College Park, Maryland 20742, pcramton@gmail.com
}

\begin{abstract}
We report on the use of a quadratic programming technique in recent and upcoming spectrum auctions in Europe. Specifically, we compute a unique point in the core that minimizes the sum of squared deviations from a reference point, for example, from the Vickrey-Clarke-Groves payments. Analyzing the Karush-Kuhn-Tucker conditions, we demonstrate that the resulting payments can be decomposed into a series of economically meaningful and equitable penalties. Furthermore, we discuss the benefits of this combinatorial auction, explore the use of alternative reserve pricing approaches in this context, and indicate the results of several hundred computational runs using CATS data.
\end{abstract}

Subject classifications: games: group decisions; bidding: auctions.

Area of review: Revenue Management.

History: Received September 2008; revisions received November 2009, July 2010, January 2011; accepted August 2011.

\section{Introduction}

Combinatorial auctions represent one of the most prominent areas of research in the intersection of operations research (OR) and economics. First proposed for practical governmental applications by Rassenti et al. (1982), a combinatorial auction (CA) is an auction for many items in which bidders submit bids on combinations of items, or packages. CAs also are referred to as "package auctions" or auctions with "package bidding." In a general CA, a bidder may submit bids on any arbitrary collection of packages. The "winner-determination problem" identifies the value maximizing assignment given the package bids. This problem is as complex as the weighted set-packing problem, and hence NP-hard (see Rothkopf et al. 1998).

Thus, in the many real-world applications of CAs, the computational techniques of OR facilitate more efficient economic outcomes in environments too complex for classical (i.e., noncomputational) economic theory. Conversely, the game-theoretic framework surrounding CAs provides a host of new computational challenges and optimization problems for OR.

One critical element of any CA is the pricing rule, which determines what each winner pays for the package won. In this paper, we present a new class of optimizationbased pricing rules for combinatorial auctions in general, demonstrate some of their unique features, and elaborate upon some properties of the larger class of core-selecting mechanisms. We also describe the use of this algorithm for recent and upcoming spectrum-license auctions in the United Kingdom, for upcoming spectrum auctions in several European countries (e.g., the Netherlands, Denmark,
Portugal, and Austria), and for use in the United States for the Federal Aviation Administration's (FAA) proposed allocation of landing rights to control congestion at airports. ${ }^{1}$ Furthermore, we provide the relevant economic interpretation and theoretical basis for our algorithm's various features.

\section{Background}

The use of auctions for allocating spectrum-license-rights to telecommunications companies gained prominence in 1994 when the Federal Communications Commission (FCC) began to use a simultaneous ascending auction (SAA) to sell spectrum licenses in the United States. The initial design, which is still used today with only slight modifications, avoided the idea of a "combinatorial" or "package" auction, in which bidders bid on packages of licenses because of the inherent computational difficulty.

A main difficulty with the SAA and other auctions that allow only bids on individual lots is the exposure problem. A bidder finds it risky to bid on a collection of lots, because of the risk of receiving an incomplete package of complements. For example, a bidder might need both $A$ and $B$. If the bidder is allowed to bid only on individual lots as in the SAA, the bidder risks winning only one of the required lots. A CA avoids this problem by letting the bidder bid on the package $\{A, B\}$ with no risk of winning just $A$ or just $B$. For a thorough discussion of the strengths and drawbacks of the SAA and its implementation by the FCC, see Cramton (2006). Also, for a general introduction to CAs, see the edited volume by Cramton et al. (2006). 
To maintain many of the strengths of the SAA while mitigating its primary weaknesses, several authors have proposed hybrid auction formats, that combine the simple price-discovery process of a "price-clock" with the efficiency and exposure-problem-elimination of a CA. (See Porter et al. 2003, Ausubel et al. 2006, and Cramton 2009.) Here we present the latest development in this line of research, a combinatorial-clock auction with quadraticcore-pricing. For the remainder of the paper, we focus on this pricing rule as adopted for several upcoming governmental auctions.

In $\S \S 2.1-2.3$ we introduce notation and a general CA model and motivate the use of core-pricing as the most usable generalization of the second-price concept from single-item auctions. In $\$ 3.1$ we describe the core of the CA game formally, and elaborate upon some of the alternative representations of the core. In $\$ 3.2$ we describe the particular core-selection rule that we proposed for the U.K. spectrum license market, describing some of its properties in $\S \S 3.3$ and 3.4. The implementation of seller reserve values is described in $\$ 4$. In $\S 5$ we briefly describe our experience implementing these pricing rules for real-life auctions, while $\$ 6$ presents results from computational experiments. Conclusions follow in $\$ 7$.

An electronic companion to this paper is available as part of the online version that can be found at http://dx.doi.org/ $10.1287 /$ opre.1110.0124. Supplementary material is provided in the electronic companion, with Appendix A.1 demonstrating how semi-sincere strategies eliminate a form of envy, A.2 and A.5 providing technical details on computational implementations, and A.3 and A.4 providing proofs of some results in the main text. The final appendix, A.6, outlines some practical considerations regarding real-life implementations.

\subsection{The Environment: Heterogeneous Goods and Bidders}

We consider an environment in which a government intends to sell many interrelated heterogeneous items. The heterogeneity of spectrum licenses arises from varying geographical coverage, as well as technological considerations, such as interference with adjacent frequency bands, etc. The primary goal of the government agency is assumed to be efficiency: the items should be sold to those who value them the most. Stated differently, the government's objective is the maximization of social welfare.

In the case of spectrum-licenses, bidders might have complex preferences over the items being auctioned, with some bidders considering certain items to be substitutes, while others treat the same items as complements. Differing technologies might give rise to such heterogeneity among the bidders' preferences. One bidder might treat any two items as substitutes because her communication technology is neutral to the spectrum on which it is transmitted, while another bidder might require a pair of adequately separated licenses as uplink and downlink frequencies for two-way communication. The latter bidder thus treats certain pairs as complements.

With a variety of new communication technologies emerging, it is important that the auction design be technology neutral. If, for example, bidders were homogeneous in their desire for "paired" licenses with a certain optimal spacing between uplink and downlink frequencies, it would be appropriate for the auction design to specify that licenses be sold as bundled pairs. If instead some bidders desire a single contiguous strip of unpaired licenses while others desire pairs, this pre-bundling of licenses into pairs would be inappropriate. This is the case for the United Kingdom's $2.6 \mathrm{GHz}$ auction, in which Ofcom determined that bidders could bid on contiguous blocks of either paired or unpaired spectrum licenses, or some combination of both; the strength of the bids themselves would determine the quantity of spectrum of each type. In general, this flexibility of package bidding, the cornerstone of CAs, provides an opportunity for OR tools to improve economic outcomes; the problem of determining the optimal set of bids to accept is generally complex and closely related to the NP-hard setpacking problem. (See Rothkopf et al. 1998 and deVries and Vohra 2003.)

Package bidding alone can often represent a daunting challenge to both the bidders and the bid-taker. In the United Kingdom's $2.6 \mathrm{GHz}$ spectrum auction, for example, there are 39 unique licenses offered for sale, and thus $2^{39}$ packages for each bidder to consider placing a bid on. In practice, the auctioneer cannot accept this full set of package bids from each bidder, so instead limits the number of package bids it will accept (in the U.K. auctions the number of bids is usually capped in the thousands). The bidders thus face the difficulty of deciding which are the "best" packages to bid on, in addition to the problem of deciding their value for any single package.

For the remainder of the paper, we therefore address the computation of prices following the final sealed-bid round in a two-stage hybrid design known as the "clock-proxy auction," as proposed by Ausubel et al. (2006). In this design a final sealed-bid CA is preceded by a preliminary "clock stage" used as a preference elicitation tool, allowing the bidders to learn about market competition and discover valuable information about which packages seem most profitable to bid on as competitive prices are revealed. We propose the use of quadratic programming in conjunction with constraint generation to determine the best set of final prices in the final sealed-bid auction of such a design, which takes all clock-stage bids and any other "supplementary" package bids made by the bidders as exclusive package offers.

\subsection{Winner Determination}

Here, we consider bidders that have participated in a clock auction (or, to be more precise, the clock-phase of a two-phase hybrid auction) and have submitted any supplementary package bids, and we consider the auctioneer's problem of determining the final set of package bids 
to accept and the payments to collect from each bidder. Although some mechanisms considered in the mechanism design literature consider the possibility of outcomes that are not efficient with respect to submitted bids (for example, Myerson 1981 or Goldberg and Hartline 2003) for the governmental allocation of public resources, we consider efficiency to be essential and indispensable in order to achieve the government's goals and to promote the perceived fairness of the auction outcome.

Let $M=\{1,2, \ldots, m\}$ represent the set of $m$ items being auctioned and $N=\{1,2, \ldots, n\}$ represent the set of $n$ bidders. Each bidder has submitted a collection of bundle bids, with $b_{j}(S)$ representing bidder $j$ 's monetary bid on any bundle $S \subseteq M$. The efficient winner determination problem over the set of bidders $N$ is defined by the following integer program, which maximizes the value of accepted bids without selling the same item to more than one bidder:

$$
\begin{aligned}
w d(N)=\max & \sum_{j \in N} \sum_{S \subseteq M} b_{j}(S) \cdot x_{j}(S), \\
\text { subject to } & \sum_{S \supseteq\{i\}} \sum_{j \in N} x_{j}(S) \leqslant 1, \quad \forall i \in M, \\
& \sum_{S \subseteq M} x_{j}(S) \leqslant 1, \quad \forall j \in N, \\
& x_{j}(S) \in\{0,1\}, \quad \forall(S, j), \text { such that } \\
& \text { a bid } b_{j}(S) \text { was submitted. }
\end{aligned}
$$

Additionally, we note that this formulation implies a specific "XOR" bidding language in which, in accordance with constraint set (2), no two bids made by the same bidder may be accepted by the auctioneer. Although a host of alternative bidding languages have been described in the literature (see Nisan 2006), we maintain this XOR formulation because it is general enough to describe any other bidding language (albeit exhaustively). Furthermore, it is this bidding language that has been used in practice in the U.K. spectrum auctions, because in general, the clock phase narrows the number of bids that will need to be bid upon substantially, keeping this formulation from growing too large, and because the implication of each bid is most easily understood by the bidders in this setting, allowing little room for confusion regarding the implication of any bid made. Each bid is an exclusive offer that cannot be recombined with any other bids of the same bidder.

\subsection{Payment Determination: Second Price Rules and Core-Selection}

We now consider the algorithm for the determination of payments in the final sealed-bid auction. First, we motivate core pricing as the appropriate generalization of the "second-price" rule.

A fundamental development of early auction theory is the equivalence (under the assumption of private values) of the outcomes in the well-known English auction (in which an item is offered at increasing prices until only one bidder continues to indicate willingness to purchase) and the second-price sealed-bid auction for a single item. Krishna (2002) provides an overview of auction theory. In the second-price sealed-bid auction, bidders submit a sealed bid for the single item being auctioned, with the highest bid winning the item and the winner paying the amount of the second highest bid. The second-price sealed-bid auction (for a single item) is well known to satisfy each of the following properties:

1. Individual rationality: each bidder expects a nonnegative payoff for participating. In the case of auctions, this simply means that nonwinners do not pay, and that each winner pays an amount less than or equal to her bid.

2. Efficiency: the highest valued bid wins. In the combinatorial case, this will be interpreted as the winning bidders form an optimal solution to (WD).

3. Dominant strategy incentive compatibility: misreporting one's value for the item(s) never gives an advantage.

4. The "core" property: no coalition (subset of all players) can form a mutually beneficial renegotiation among themselves. In the case of an auction, this simply means that the seller would not prefer to ignore the outcome dictated by the auction and renegotiate with a subset of the bidders.

It is also well known that the Vickrey auction, also known as the Vickrey-Clarke-Groves or VCG mechanism, is the unique mechanism in the combinatorial setting that satisfies properties 1,2 , and 3 from this list. The VCG outcome implements the efficient solution described by (WD), and each winning bidder $j$ receives a discount from her winning bid amount, equal to $w d(N)-w d(N \backslash\{j\})$, which induces her to bid honestly. Unfortunately, it is easily shown that property 4 does not hold for the VCG mechanism. The reader may easily verify that in a two item auction for items $A$, and $B$, with bids by three bidders $b_{1}(A)=2, b_{2}(B)=2, b_{3}(A, B)=2$, the VCG payments are both zero for winning bidders 1 and 2 , despite a competing bid of 2 on the items they win. Thus this simple example (from Ausubel and Milgrom 2002) illustrates that the core property is not upheld by the VCG auction, because both the seller and bidder 3 would prefer to renegotiate for both items at any price in the open interval $(0,2)$.

Given the beauty of the VCG mechanism in its ability to elicit truthful revelations of preferences from the bidders, it is not surprising that it has received a great amount of attention in the literature. However, several authors have noted that the VCG auction is not practical for actual implementation. (The reader may refer to Rothkopf 2007, Ausubel and Milgrom 2006, or Rothkopf et al. 1990 for example.) We instead contribute to the growing literature that "core-selecting mechanisms" or "auctions with core pricing" provide the most usable generalization of the second-price sealed-bid auction paradigm to the combinatorial setting. In this category of CAs, we eschew the approach of the VCG mechanism (which treats 
properties 1-3 as constraints and ignores property 4) and instead treat properties 1,2 , and 4 as constraints while minimizing (with respect to some metric) the deviation from property 3 . We do not, however, completely disregard the VCG outcome, but instead use it as a baseline for incentive compatibility; the closer we get to the VCG payments, the incentives to distort one's bids become less and less. Furthermore, when the VCG mechanism does happen to satisfy property 4 , our mechanism will also produce the VCG outcome.

A core-selecting mechanism is one that satisfies property 4 when bids are treated as true values for the corresponding bundles. (This distinction about true values is necessary, because property 3 does not hold in general, so we cannot claim that bids are equal to true values.) Before delving into the technical description of how we propose to compute core prices in the aforementioned governmental auction applications, we now briefly summarize some of the properties of core selecting mechanisms which motivate our claim that bidder-optimal core prices provide a useful notion of second prices for the combinatorial setting.

To begin, for any core-selecting mechanism:

- An allocation must be efficient with respect to reported preferences (see Milgrom 2004).

- No bidder can ever earn more than her payoff under the VCG auction by disaggregating and using false-name or shill-bidders (see Day and Milgrom 2008). Note that this is not true of the VCG auction.

- Determining a core outcome is NP-hard whenever the winner-determination problem is NP-hard (see Day and Raghavan 2007).

- For any profile of opponents' bids, each bidder has a best reply that is a semi-sincere strategy, i.e., given true utility $u_{j}(S)$ for each item set $S$, each bidder $j$ has a best strategy of the form $b_{j}(S)=\max \left(0, u_{j}(S)-\alpha_{j}\right)$ with the same $\alpha_{j} \geqslant 0$ for each bundle $S \subseteq M$ (see Day and Milgrom 2008).

This last point says that there exists a semi-sincere strategy among any bidder's set of optimal strategies (so-called as a bidder is truthful about the relative values of bundles receiving positive bids), which is elsewhere referred to alternatively as either a truncation strategy (by analogy to truncation strategies in matching markets) or a profittarget strategy (as a bidder $j$ targets an amount of profit $\alpha_{j}$ and cannot receive less than this amount of profit when among the winning bidders). In Appendix A.1, we provide further motivation for the use of semi-sincere strategies in core selecting auctions, in their ability to eliminate ex post envy. Similar connections between envy reduction and core-selection are provided in the concurrent work of Othman and Sandholm (2010).

Next, we consider bidder-optimal core mechanisms, which are optimal, or efficient, in the Pareto sense. That is, if the auction determines an efficient allocation and prescribes payment vector $p$, then there is no alternative payment vector $p^{\prime}$ also in the core, such that $p^{\prime} \leqslant p$.
As is typical in Pareto-optimality, this can be read as: no bidder can be made better off without another being made worse off. If the core-selecting mechanism is also bidderoptimal, we have the following:

- The incentives to unilaterally misreport are not dominated by any other core-selecting mechanism (see Day and Milgrom 2008).

- If the buyer-submodularity condition holds, then the Vickrey outcome is the unique bidder-optimal core point. Thus any bidder-optimal core-selecting mechanism is equivalent to the Vickrey mechanism whenever bids and valuations satisfy buyer-submodularity, in which case the auction is dominant-strategy incentive-compatible. The same result holds if the more restrictive gross-substitutes condition is satisfied for bids and valuations (see Ausubel and Milgrom 2006). Furthermore, if it is common knowledge that the buyer-submodularity condition holds (for valuations) then truth-telling by all bidders is a Nash equilibrium in any core-selecting auction, even if bidders are free to use shill bidders. (This follows from Theorem 1 of Day and Milgrom 2008, which implies that any player's payoff is no more than her induced Vickrey payoff in any core-selecting auction, even if using shills.)

- Any bidder-optimal core payoff vector induces a semisincere strategy that is a full-information Nash equilibrium (see Day and Milgrom 2008 or Day and Raghavan 2007).

- Any Nash equilibrium in which winners use semisincere strategies and losers bid truthfully achieves a bidder-optimal core point with respect to the true valuations of the bidders. Thus at any such full-information equilibrium an outcome must be efficient with respect to true preferences, not just relative to reported preferences/bids (see Day and Milgrom 2008 or Day and Raghavan 2007).

These last two points elucidate what might be seen as a "strategic correction" property of bidder-optimal coreselecting auctions. Bernheim and Whinston (1986) show a similar theorem that the bidder-optimal core points are precisely the full-information Nash equilibria in a first-price (i.e., pay-as-bid) format. Thus in a core-selecting auction, if all bidders bid truthfully, the auction makes them pay an amount equal to what they "should have bid" in a first-price format, effectively correcting their strategies. By analogy, the second-price sealed-bid auction corrects the winner's bid to what she should have bid to just tie the bid of the next highest bidder, if she had known how much that was. The main difference in the combinatorial setting is that there are many bidder-optimal outcomes and thus many equilibrium strategies, so the auction additionally helps the bidders by selecting an equilibrium to coordinate to. In this paper, we explore various attractive criteria for selecting such an equilibrium outcome.

This point regarding strategic correction is worth emphasizing for its relevance in response to regulators worried about the adoption of a core-selecting auction, who may ask (as they did at the FAA when adopting a core-selecting rule), "Aren't the core-prices overly complicated? Wouldn't 
the bidders prefer the simplicity of a pay-as-bid bid rule?" The results on the Nash equilibria in these auctions allows us to answer that in fact the opposite is true. In a firstprice auction, it is difficult to determine the correct bidding strategy which will result in payments as small as possible fixing the other bidders' bids, and every dollar bid above this optimal amount is a dollar wasted. However, in a core-selecting auction, the auctioneer effectively corrects the bids for you so that at the conclusion, having seen the payments of the other bidders, each bidder would agree to bid exactly her payment amount, given that all other bidders bid exactly their payment. Furthermore, this is exactly what they would have liked to have bid as a group in a pay-as-bid auction. This point will be further illustrated numerically with Figure 1 and Example 1, provided in the following section.

\section{Selecting a Core Outcome}

As noted in $\$ 2.3$, the bidder-optimal points in the core represent satisfactory outcomes from the auction. Bidders are satisfied that they are just paying enough to beat out competitors, and that no one can be made better off without another being made worse off. The seller is satisfied to receive competitive revenues determined by competition and for which no readily apparent better alternative is available. We now define the core formally, noting a few interesting alternative formulations and their uses, and demonstrate some new techniques for selection among core outcomes.

In addition to notation already introduced, let payment vector $p \in R_{+}^{n}$ represent the nonnegative vector of payments for each bidder, and let $\pi_{j}=b_{j}\left(S_{j}\right)-p_{j}$ represent the observable surplus or profit experienced by bidder $j$ when the auction awards bidder $j$ set $S_{j}$. Bidders are said in this case to have quasi-linear utility (in that their profit is linear in payment). Also, one may note that we are dealing only

Figure 1. The core point closest to VCG payments.

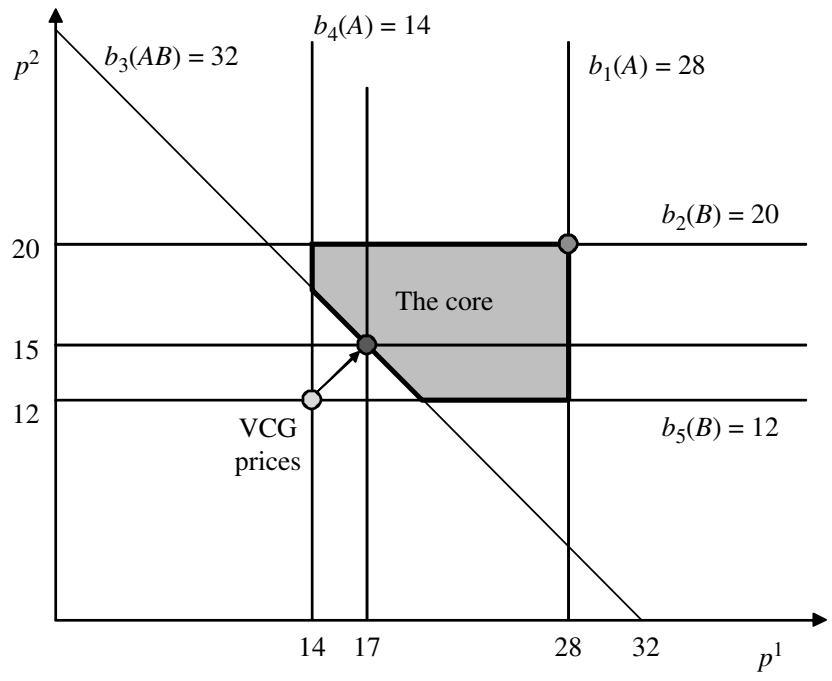

with observable surplus, not true net utility $u_{j}\left(S_{j}\right)-p_{j}$, because without a guarantee of incentive compatibility, the auctioneer will have no knowledge of these amounts. Also, we may write $\pi_{0}=\sum_{j \in N} p_{j}$ for the profit of the seller. (For now, we assume that prices are normalized by reserve amounts, so that we need not subtract the value of each item from the seller's profit. Stated differently, the seller has no value for keeping the items herself.)

An outcome is represented by a feasible solution to problem (WD), which we will specify by the set of awarded (possibly empty) bundles $\left\{S_{j}\right\}$ for each bidder and a payment vector $p$, thus inducing a profit vector $\pi$. An outcome is said to be blocked by coalition $C \subseteq N$ if there is some alternative outcome with awarded bundles $\left\{\bar{S}_{j}\right\}$ and payments $\bar{p}$, such that $\bar{\pi}_{j}=b_{j}\left(\bar{S}_{j}\right)-\bar{p}_{j} \geqslant \pi_{j}$ for all $j \in C$, and for which $\bar{\pi}_{0}=\sum_{j \in C} \bar{p}_{j}>\pi_{0}$. An outcome that is not blocked in this context is said to be in the core with respect to the submitted bids $b$. For this paper, we may simply say that the outcome is in the core, because we do not consider the underlying utility functions. Also, because in other economical settings the core is not always guaranteed to exist, it is worth noting that in this setting the pay-what-you-bid point is always in the core, and thus the core is always nonempty.

It might be helpful at this point to consider an example. Let $m=2$ items, $A$ and $B, n=5$ bidders, and let bids be as follows (each bidder submits only one bid).

EXAMPLE 1.

$$
\begin{array}{ll}
b_{1}(A)=28, & b_{2}(B)=20, \\
b_{3}(A B)=32, & b_{4}(A)=14, \quad b_{5}(B)=12 .
\end{array}
$$

It is easy to determine that the unique winners in the efficient allocation are bidders 1 and 2 and that the VCG payments are $p_{1}^{\mathrm{VCG}}=14$ and $p_{2}^{\mathrm{VCG}}=12$. The core itself can be graphed in payment space as in Figure 1.

Here we note that due to the simplicity of the example, the constraints defining the core are simply the bids of the losing bidders (this is not always the case). In particular, because bidder 4 would always object (block) if bidder 1 paid less than 14 for item $A$, we have the constraint $p_{1} \geqslant 14$. Similarly, bidder 5 dictates $p_{2} \geqslant 12$. Bidder 3 would object if bidders 1 and 2 together did not beat his bid on the items they have won, thus $p_{1}+p_{2} \geqslant 32$. Upper bounds on payments are given by the bids themselves, consistent with our assumption of individual rationality. Next, one will note from the picture that we are in a situation for which the VCG outcome is not in the core; bidder 3 alone forms a blocking coalition.

Using the technique of Day and Raghavan (2007), one can guarantee bidder-optimality by minimizing total payments over the core, so for Example 1 we could determine any payment vector on the line segment connecting the point $(14,18)$ to $(20,12)$, any of which represents a bidder-optimal core point. This simple example also clearly 
illustrates the statements from $\$ 2.3$ regarding Nash equilibria in a first-price auction. Fixing the bid $b_{2}$ of bidder 2 anywhere in the range $[12,18]$, bidder 1's optimal strategy in the first-price auction is to bid $32-b_{2}$, and conversely, fixing $b_{1}$ within the range $[14,20]$, bidder 2 's best bid is $32-b_{1}$. In practice, however, each bidder will not know the bid of the other, making coordination to any one of these points difficult if not impossible. Thus a risk-averse bidder would typically have to bid more than the optimal amount (i.e., where he would bid if he knew what the other were bidding). In the first-price auction, this problem, caused by lack of information, costs the bidder in a one-to-one fashion. From bidder 1's perspective, every dollar bid over $32-b_{2}$ is a dollar wasted. In any core-selecting auction, on the other hand, the bidders need only bid somewhere within the core (including bidding truthfully) and the auction will charge them a total of 32 .

But still, there is a lack of precision because the Day and Raghavan (2007) algorithm does not specify which of these bidder-optimal points should be chosen. We are motivated, however, by the observation of Parkes et al. (2001), that the difference between a final payment and the VCG payment represents a measure of "residual incentive to misreport," and so should be minimized. As one method to achieve a simple to compute minimization of the groups' incentive to deviate from truth-telling, we propose the following refinement of the Day and Raghavan (2007) procedure: over all total-payment minimizing core points, select the one that minimizes the sum of square deviations from the VCG payment point. Of course, minimization of this amount is equivalent to minimizing the positive square root of this amount, so one may rightly describe this selection as the core point with minimum Euclidean distance from VCG. This rule can be referred to as a VCG-nearest or Vickreynearest rule.

For Example 1, this results in the unique payment outcome $(17,15)$. Interestingly, this outcome is unchanged (for Example 1) as long as bidder 1 bids at least 20 and bidder 2 bids at least 18, fixing the bids of the losing bidders. The values 20 and 18 are the minimum amounts that could be bid, respectively, without the bid of 32 emerging in the VCG computations. If bidders 1 and 2 bid more than 32 in total, and less than 20 and 18, respectively, than the VCG point can in fact move, causing a slightly different outcome. For example, if the bids for 1 and 2 changed to 19 and 16 , the VCG point shifts to $(16,13)$, and final payments become $(17.5,14.5)$. Thus, when the VCG point moves, the relative payments can also change slightly, but the payoff to the seller remains unchanged.

One may also note that bidders 1 and 2 in Example 1 each pay an equal amount ( 3 units) above their VCG payments in order to match the blocking bid made by bidder 3. This is indeed a general phenomenon (based on the Karush-Kuhn-Tucker optimality conditions) and one that we describe in detail in \$3.4. First, however, we describe a few distinct presentations of the core and the interesting implications/economic interpretations of each formulation.

\subsection{Core Formulations}

First, working straight from the definition, the coalitional core constraints are most commonly modeled (in the economics literature) as in Day and Milgrom (2008):

$\sum_{j \in C \cup 0} \pi_{j} \geqslant w d(C) \quad \forall C \subseteq N$,

emphasizing that final payoffs (on the left) must exceed the value that each coalition $C$ can generate if they alone deal with the seller (on the right). Yet from the point of view of computation, this formulation hides the (discrete) selection of a bundle for each bidder, and is thus not guaranteed to be convex in $\pi$-space, making it a difficult formulation for use in a direct computational implementation. In practice, we take a divide-and-conquer approach, first solving the winner-determination problem and then computing core payments once a particular set of winning bundles $\left\{S_{j}\right\}$ has been determined. Substituting in these bundles, canceling payments that are duplicated in the $\pi_{0}$ term, and recognizing that $w d(N)=\sum_{j \in N} b_{j}\left(S_{j}\right)$ yields an alternative formulation:

$\sum_{j \in W} p_{j} \geqslant w d(C)-\sum_{j \in C}\left(b_{j}\left(S_{j}\right)-p_{j}\right) \quad \forall C \subseteq N$,

where $W$ represents the set of bidders who win nonempty bundles. Here the right-hand side reflects what coalition $C$ is willing to offer to the seller at payment vector $p$; they will offer as much as can be obtained from them as a group, $w d(C)$, less the profit they are already making at payment vector $p$, which is $\sum_{j \in C}\left(b_{j}\left(S_{j}\right)-p_{j}\right)$.

As shown by Day and Raghavan (2007), this formulation is convenient from an algorithmic point-of-view, when we treat computations modularly (i.e., with a blackbox mindset). If we already have code (a blackbox) that solves winner-determination problems, and we are considering whether a particular payment vector $p$ is in the core, we can simply reduce each bid by the surplus at $p$ and re-run the winner determination. This will find the coalition making the highest offer to the seller and if this is more than the current total payments, then a violated core constraint has been identified. (In fact, this finds the most violated constraint.) This complexity equivalence between separation and winner determination is helpful to demonstrate that finding core outcomes is indeed of equivalent complexity as winner determination (see Day and Raghavan 2007). Also, this formulation is noteworthy because it is in this form that the "core" was defined legally within the regulations for the U.K. spectrum auctions. Rather than defining the core in terms of possible renegotiations, this separation formulation gives a clearly defined, mechanically checkable stopping criterion, or provides a certificate that a payment vector is not in the core.

Finally, from a math programming standpoint, it is most helpful to segregate decision variables and constants on their respective sides of the inequality. This yields the following formulation, which follows from the previous 
formulation by simply canceling payment terms appearing on both sides:

$\sum_{j \in W \backslash C} p_{j} \geqslant w d(C)-\sum_{j \in C} b_{j}\left(S_{j}\right) \quad \forall C \subseteq N$.

We use this formulation for our actual computations of core prices, which we find by quadratic optimization over the core. This formulation, too, has its own interesting economic interpretation, lending further credence to coremechanisms as selecting "fair" payments. Given the efficient allocation $\left\{S_{j}\right\}$, the right-hand side of (6) finds what coalition $C$ would pay to get everything, $w d(C)$, minus what they would pay for what they actually get and is thus equal to the most coalition $C$ would be willing to pay to take away what the complementary set of bidders is getting. Thus each core constraint says that any set of bidders pays at least as much as their opponents would pay to take their stuff away from them, a competitively pleasing and arguably fair proposition indeed.

\subsection{Quadratic Rules for Payment Determination}

Letting $\beta_{C}=w d(C)-\sum_{j \in C} b_{j}\left(S_{j}\right)$, and denoting the vector of all such $\beta_{C}$ values as $\beta$, formulation (6) can be written more compactly as

$p A \geqslant \beta$,

where each column $a_{C}$ is the characteristic vector of the complementary set of winners. (That is, the $j$ th entry in $a_{C}$ equals 0 if bidder $j$ is in set $C$ and equals 1 if bidder $j$ is not in $C$. Because nonwinners never pay, the dimension of each $a_{C}$ is $|W| \times 1$, rather than $n \times 1$.) The core-selection region is defined by these constraints as well as the individual rationality constraints: $p \leqslant b$, where each component $b_{j}$ in the vector $b$ is given by $b_{j}=b_{j}\left(S_{j}\right)$.

We now present a class of algorithms for core-selection based on quadratic programming. Motivated by the concurrent work of Erdil and Klemperer (2009), these rules can be referred to as reference rules, in which payments are determined by minimizing the Euclidean distance to a reference vector of prices. A $p^{0}$-reference rule finds final payments $p^{*}$ that minimize the sum of squared deviations from payment reference point $p^{0}$, which might be either constant or dynamically determined but is constant with respect to the following optimization:

$\min \left(p-p^{0}\right)\left(p-p^{0}\right)^{T}$,

$p A \geqslant \beta$,

$p \leqslant b$.

Also, Day and Raghavan (2007) provided some motivation that payment minimization over the core may deter certain types of group deviation and that a threshold rule (as described by Parkes et al. 2001) without explicit payment minimization might not result in payment minimization. Similarly, a reference rule as just described might not minimize total payments over the core, unless this payment minimization is enforced explicitly. (Example 2 on page 34 provides an example of this phenomenon.) We therefore also describe $M R C$-reference rules in which the feasible set of payments is limited to those core points that minimize total revenue, referred to as the minimum revenue core or MRC by Erdil and Klemperer (2009). To employ such a rule, we first find minimal core payments by solving the LP:

$\mu=\min p 1$,

$p A \geqslant \beta$,

$p \leqslant b$.

Then determine final payments $p^{*}$ as the optimal solution to the following QP:

$\min \left(p-p^{0}\right)\left(p-p^{0}\right)^{T}$,

$p A \geqslant \beta$,

$p \leqslant b$,

$p 1=\mu$.

This last MRC-reference rule with $p^{0}=p^{\mathrm{VCG}}$ is the auction format adopted by Ofcom for spectrum license auctions in the United Kingdom.

In practice, evaluating each $\beta_{C}$ requires the solution of a winner-determination problem, so with $2^{n}-1$ nonempty coalitions to consider, it is advantageous to employ a coreconstraint-generation procedure as in Day and Raghavan (2007), which we henceforth abbreviate CCG. Starting at the payment vector $p^{0}$, reduce each bid by the current surplus, i.e., for all $S \subseteq M$, let $b_{j}(S)=\hat{b}_{j}(S)-b_{j}\left(S_{j}\right)+p_{j}^{0}$, where $\hat{b}$ represents the fixed, submitted bid. Then solve (WD) with these new bids, finding the first violated coalition $C_{1}$, the set of bidders winning nonempty bundles in this altered version of (WD). We then let our first approximation of matrix $A$ be simply $A_{1}=a_{C_{1}}$, and let $\beta^{1}=\beta_{C_{1}}$. Next we solve formulation (LP) with $A_{1}$ and $\beta^{1}$ replacing $A$ and $\beta$, yielding minimum payment solution $\mu_{1}$, and then solve formulation (QP) with $A_{1}, \beta^{1}$, and $\mu_{1}$ replacing $A, \beta$, and $\mu$, labeling the solution to (QP) as $p^{1}$. The algorithm continues in this fashion, finding a new violated constraint $p a_{C_{t}} \geqslant \beta_{C_{t}}$ at $p^{t-1}$ and concatenating the corresponding column to $A_{t-1}$ and new entry to $\beta^{t-1}$, forming $A_{t}$ and $\beta^{t}$, as long as this solution to the surplus-reduced (WD) exceeds $p^{t-1} 1$. If the solution to the surplus-reduced (WD) does not exceed $p^{t-1} 1$, then we may set $p^{*}=p^{t-1}$ and terminate with a solution to $(\mathrm{QP})$, representing final payments in the auction. Further discussion on the efficacy of this CCG approach is given in Appendix A.2. 


\subsection{Robustness and Constant Reference Rules}

It has been observed by Ott (2009) and Lamy (2010) that revenues might in some cases decrease when bids increase in any bidder-optimal core-selecting auction, contradicting an erroneous proposition put forth in Day and Milgrom (2008). Here we provide a result regarding the relative insensitivity of the auction outcome (including total revenues of the seller) under a special set of assumptions, helping to motivate the use of certain reference rules, and provide further insight into the types of strategies that lead to profitable deviation from truth-telling.

THEOREM 1. Consider a reference rule (or an MRCreference rule) in which the reference vector $p^{0}$ is independent of winners' bids. Suppose that bidders restrict to semi-sincere strategies and that for a fixed set of bids, the individual rationality constraints are never binding, i.e., suppose that $p^{*}<b$. Then, the auction outcome does not change for any uniform increase in a semi-sincere strategy made by a winning bidder.

Proof. See Appendix A.3.

Although the exploration of appropriate reference vectors that satisfy the condition of independence from winners' bids remains open, it is easy to see that a constant reference vector selected in advance by the auctioneer would be independent, satisfying that hypothesis of the theorem. Interestingly, this theorem states that if a bidder focuses on semi-sincere strategies (perhaps to relieve envy possibilities via Lemma 7.2), then if she bids enough that she will have paid more than she had bid, she might as well have bid honestly.

Also, this theorem can also be stated "locally" for a single winning bidder, if $p^{0}$ does not change with a uniform increase in semi-sincere strategy by bidder $j \in W$, and if $p_{j}^{*}<b_{j}$, then the overall auction outcome does not change with any uniform increase in semi-sincere bidding strategy by bidder $j$. Indeed, this local result applies under a VCGnearest rule when it is the case that $p_{j}^{*}<b_{j}$ and that bidder $j$ participates in the efficient solution even when any other single bidder is removed. In that case, each VCG payment for a bidder $\bar{j} \neq j$ remains unchanged following the semisincere increase via cancellation of the increase, and thus $p^{0}$ is unaffected by the increase. The reader may verify that this is the case for Example 1, that any bid increase by a winning bidder leaves the outcome unchanged.

But of course, the hypothesis that $p^{*}<b$ is indeed a strong assumption, and considering a violation of such an assumption elucidates situations in which there is scope for profitable deviation from truth-telling. For example, let us revisit Example 1 from page 592, but now consider a situation in which rather than a dynamic VCG-nearest rule, the auctioneer had arbitrarily selected the point $(14,12)$ as $p^{0}$, resulting in a confirmation of the independence assumption. (The following explanation would change little if we used another constant vector as $p^{0}$.) We see that the outcome of the auction is unchanged as long as bidders 1 and 2 bid any amount greater than or equal to $(17,15)$, respectively, on their bundles of interest. (Notice that any bids less than or equal to value on the bundle of interest constitute semi-sincere strategies when bidders are single-minded.) The scope for profitable bid-shading occurs only when one of the bidders bids below her final payment under truthtelling, and only when the other winning bidder bids at least as much in the other direction, strictly above her own final payment. For example, if bidder 1 knows that bidder 2 will bid 19, she can bid any amount down to 14, which (assuming preference in a tie-breaking rule) results in no change of allocation. (We see that in addition to being sure that bidder 2 bids enough to make the sum of their bids exceed 32 , bidder 1 would also like to be sure to beat the competing bid of bidder 4.) If this new bid amount for bidder 1 is less than 17 (her payment if she were honest), she will pay exactly as bid, violating the assumption of Theorem 1, and bidder 2 will be forced to pick up the difference $32-b_{1}$. Of course, without knowledge of bidder 2 's bid and bidder 4's bid, this shading below 17, which is profitable, also carries the risk of missing the efficient allocation, resulting in zero payoff.

This example shows the limitations of Theorem 1, but because a bidder $j$ will often not have enough knowledge to safely shade to a point where $p_{j}^{*}=b_{j}\left(S_{j}\right)$, the theorem is likely to be relevant in many situations. Roughly speaking, if we consider pay-as-bid outcomes to be unlikely, then this theorem states that decreases in $\alpha_{j}$ to reduce risk will likely not be costly. Also, this alteration of Example 1 seems to suggest that the most attractive combinatorial auction would be one in which the auctioneer knew the VCG point based on true valuations and used this true VCG point as the reference price vector $p^{0}$. But it is hard to imagine a situation in which the auctioneer would have enough foreknowledge to predict the true VCG point accurately yet still feel the need to conduct an auction.

Still, this motivates the goal of the auctioneer conducting a reference rule auction, to attempt to select an independent reference point that approximates the true VCG point as well as possible, in an effort to maintain the desired notion of "near-truthfulness." Prior to the influence of Erdil and Klemperer (2009), we did consider constant- $p^{0}$-reference rules, but rejected this idea in our consultation to Ofcom for U.K. spectrum auctions due to the distortions caused in the final payments, favoring large bidders, and because this approach makes the final distribution of payments highly dependent on the assumptions and actions of the auctioneer. Let us elaborate on these points, again by example.

It is easy to see with a two-winner auction that a constant reference rule, such as $p^{0}=\overrightarrow{0}$, favors larger bidders. If we were to apply such a rule to the data for Example 1, for example, bidders 1 and 2 both pay 16, an equalization of payments despite higher marginal competition on item $\mathrm{A}$ from bidder 4 . The following more extreme example demonstrates how this problem can get worse as the situation becomes more lopsided. 
EXAMPLE 3.

$$
\begin{array}{ll}
b_{1}(A)=100, & b_{2}(B)=20, \\
b_{3}(A B)=60, & b_{4}(A)=50 .
\end{array}
$$

In this example, which is similar to Example 1, the VCG payments for efficient winners 1 and 2 of $(50,0)$ are not in the core; the two must raise their combined payments to 60 in order to keep bidder 3 from blocking. If the reference rule $p^{0}=0$ is used, the result is that bidder 2 will pay the entire burden of this total payment increase; final payments become $(50,10)$, while a VCG-nearest rule (MRC or not) results in a sharing of this burden, with payments $(55,5)$. This problem can be mitigated by using a more sophisticated constant value (for example, selecting the reference vector with each term set equal to the sum of the reserves values for each item in the respective winning bundles), but the problem persists that bundles with high value relative to the auctioneer's expectations pay less of the burden of overcoming a competing coalitional offer. Because the underlying assumption of an auction is often that the seller has poor a priori knowledge of value relative to that of the bidders, we are motivated to select an outcome based on good (bid-based) information over poor (seller prior) information.

Furthermore, a one-for-one change on the part of the seller often results in a corresponding one-for-one change in payments under a constant reference rule, making the outcomes highly sensitive to the pre-auction actions of the seller. For example, considering Example 1 with a sellerpredefined constant reference rule $p^{0}=(14,12)$, we end at the payment vector $p^{*}=(17,15)$. But if the seller had instead selected the reference vector $p^{0}=(15,11)$, a one-for-one change, the resulting payment vector is $p^{*}=$ $(18,14)$, a corresponding one-for-one change. This sensitivity puts a great deal of pressure on the auctioneer in the selection of the constant reference point and opens the possibility of post-auction lawsuits if the criteria for the reference point selection cannot be adequately justified. A zero-reserve (or a bound-only reserve, as will be discussed in §4) VCG-nearest rule, however, does not suffer from this sensitivity to auctioneer selection and could therefore be seen as a safer design choice on the part of the seller.

\subsection{The Karush-Kuhn-Tucker Optimality Conditions}

Employing a typical tool from the nonlinear programming toolkit (see for example Bazaraa et al. 1979) we derive the Karush-Kuhn-Tucker (KKT) conditions for the optimality of problem $(\mathrm{QP}) .{ }^{2}$ These conditions are necessary and sufficient because the constraint-defining functions are linear (hence quasiconvex) and the objective is convex, as long as the reference point $p^{0} \leqslant p$ for all $p$ in the core. ${ }^{3}$ Letting $\tilde{A}$ be the submatrix of $A$ consisting of the columns that are tight at $p$, then the KKT conditions indicate that $p$ is an optimal solution to (QP) if and only if there exist a vector $z \geqslant 0$, a vector $w \geqslant 0$, and a scalar $v \geqslant 0$, such that

$p=p^{0}+z \tilde{A}^{T}-v \overrightarrow{1}-w I_{p}$,

where the matrix $I_{p}$ contains a row of the identity matrix $e_{j}$ for each bidder $j$ who pays as bid at $p$.

Thus the final payment vector $p^{*}$ can be decomposed as follows for each bidder:

$p_{j}^{*}=p_{j}^{0}+\sum_{a_{C} \in \tilde{A} \mid j \notin C} z_{C}-v-w_{j}$,

that is, each bidder $j$ pays her VCG value plus a penalty for any marginally unblocking (i.e., tight) coalition $C$ that $j$ does not belong to (and this penalty is equal for all bidders not in $C$ ), minus an offset term $v$ that is equal across all bidders and serves to guarantee payment minimization, and minus a personal offset term $w_{j}$ to guarantee individual rationality for a pay-as-bid bidder $j$. The equity of these $z$ terms across bidders contributes to the "fairness" of this payment rule; payments are based on equal contributions to overcome a competitive challenge from other bidders, except where individual rationality constraints cap the contributions of a bidder, in which case the personalized offset $w_{j}$ takes affect. If a non-MRC-reference rule is employed, then the $v$ terms disappear from this decomposition, as the relevant constraint disappears from the derivation of these KKT conditions. Furthermore, we have the following lemma.

Lemma 3.1. Under a VCG-nearest rule, $w_{j}=0$ for all $j \in N$.

Proof. See Appendix A.4.

Thus a non-MRC VCG-nearest rule provides the simplest payment decomposition. Winners only pay equal penalties for a coalition $C$ they do not belong to, $z_{C}$, with no universal adjustment $v$ and no personalized adjustment $w_{j}$.

If one were to consider using the same feasible region but a different strictly convex objective function, the KKT derivation changes only in the objective gradient terms. So strictly speaking, with a new objective function $f\left(p-p^{0}\right)$, rather than a linear decomposition of $p-p^{0}$, we get a linear decomposition $\nabla f\left(p-p^{0}\right)=z \tilde{A}^{T}-w I_{p}-v 1$. So if we instead minimized $\sum_{j \in W}\left(p_{j}-p_{j}^{0}\right)^{4}$, we would have for each bidder a linear decomposition of $\left(p_{j}^{*}-p_{j}^{0}\right)^{3}$, seeming only to add confusion to the breakdown of payments and further motivating the quadratic objective as the most simple convex objective function to interpret.

It is also worth noting that the optimal solution $p^{*}$ to $(\mathrm{QP})$ is unique because we are minimizing an $\mathrm{L}_{2}$-distance to a fixed point over the convex set of payment minimizing core points (and if there were multiple optima, the triangle inequality would verify that a convex combination of these "optima" had a lower objective value, a contradiction). But the vector $(v, w, z)$ on the other hand, which 
decomposes these payments, might not be unique. We elaborate upon this phenomenon in Appendix A.5. Also, the KKT conditions allow us to identify a quick solution technique for solving (QP) at intermediate stages of the constraint generation algorithm, which we also elaborate upon in Appendix A.5.

\section{Seller Reserves}

Here we consider the subtleties of applying the quadratic core-selection approach when the seller has a nonzero reserve value for some or all of the items being sold. For example, in many single-item auction environments, reserve prices from the seller are easily modeled by simply having the seller submit a "dummy" bid equal to the reserve amount on the item; if the seller wins, then the item is kept. Here we note that this approach might be misapplied in the context of a core-selecting auction if the proper care is not taken.

Let us consider a seller who has an additive reserve value $r_{i}$ for each item $i$ in the auction, or collectively, the seller has a reserve vector $r$, which will be treated additively. That is, the seller's net payoff is given by $\pi_{0}=$ $\sum_{j \in W} p_{j}-r \cdot x$, where $x$ is the characteristic vector of the items sold. Letting $r_{S}=\sum_{i \in S} r_{i}$ for any $S \subseteq M$, the socialwelfare-maximizing objective of the winner determination problem then becomes

$w d(N)=\max \sum_{j \in N} \sum_{S \subseteq M}\left[b_{j}(S) \cdot x_{j}(S)\right]-r \cdot x$,

which is in turn equivalent to

$\max \sum_{j \in N} \sum_{S \subseteq M}\left[b_{j}(S)-r_{S}\right] \cdot x_{j}(S)$.

Thus as is standard when considering VCG mechanisms with reserves (see, for example, Ausubel and Cramton 2003), the auction outcome can be computed by first reducing each package bid by the total reserve amount for the package, then proceeding as if the seller had zero reserves, and finally adding the bundle reserve back in to determine final payments.

Consider the following simple two-bidder, two-item example.

$\operatorname{ExAmPlE}^{4}$ 4. $b_{1}(A)=40, b_{2}(A B)=40$, and the seller has a reserve value of 10 for each item.

Efficiency demands that item $A$ be sold to bidder 1, while $B$ remains unsold. With the reduction of bids approach, we may begin by reducing each bid by the total reserve amount for the package, resulting in reduced bids, $b_{1}^{r}(A)=30$ and $b_{2}^{r}(A B)=20$. We then compute a VCG payment of 20 for bidder 1 , and applying CCG, we note that bidder 2 does not block this VCG outcome. These reserve amounts must be added back into any final payment to produce the actual final payment for bidder $1, p_{1}=20+10=30$. (Notice that if $p_{1}=20$, the seller and bidder 2 would prefer an exchange of both items for any price in the open interval $(30,40)$. Also note that if the seller instead had a zero reserve value, either bid could be accepted as an efficient solution, and in either case the winner would pay 40.)

This procedure might seem to contain a redundancy, given that we first reduce each package bid by the reserve amount for the package, and then add this package reserve amount back into any final payment. Given that seller reserves are adequately modelled using "dummy bids" in the context of single-item auctions, for example, one might be tempted to try the following approach: leave bids in their "unreduced" form, treating the seller as if his reserve value was zero, and insert a "dummy bidder" or "reserve bidder" to represent the interests of the seller, bidding the reserve amount for each item. In this example, we would add bids $b_{3}(A)=10$ and $b_{4}(B)=10$. This approach does necessarily lead to the correct determination of the efficient solution; the bids made by 1 and 4 win, with a winning reserve bidder indicating that item $B$ stays with the seller. But a naïve application of the CCG algorithm proceeds as follows: following determination of the efficient solution, VCG payments are computed for bidder 1 and (dummy) bidder 4 as $p_{1}=30, p_{4}=0$. But this set of payments is blocked by bidder 2, who would be willing to pay up to 40 to take both items away from the winners. Minimizing the distance to the VCG point (for example) after applying the relevant core constraint payments are adjusted to $p_{1}=35, p_{4}=5$, which is in the core with respect to these four bids, given that the dummy bidders are treated just as any other bidder.

But this treatment of the dummy bidders just as any other bidder is at the heart of the problem, and as we can see, this misapplication causes bidder 1 to pay more. Although the determination of the efficient solution proceeds correctly when the seller's reserve amount is replaced with a sellerdummy in the objective of the WDP, the surplus reduction step in the generation of core constraints proceeds incorrectly; it fails to consider that the seller loses the value of item $B$ if it were to be reallocated to bidder 2 , thus overstating the seller's willingness to form a blocking coalition with bidder 2 .

But the use of seller-dummy bidders is intuitively appealing; the seller wants to leave open the possibility of buying back some of its own property if competition is too low, and wishes the competition for its own property to be reflected both in the determination of winners and in payments. An easy fix is available that maintains this intuitively appealing use of dummy bidders to reflect reserves, however, and it is this variation which was used in the rules published as part of the December 2008 FAA slot-auction bidder seminar. Those rules included the following (paraphrased) treatment of seller reserves:

- The seller will specify a reserve amount for each item, $r_{i}$, stipulating that any bid $b_{j}(S)$ must not be less than $r_{S}$ and that any payment made by any bidder for package $S$ must not be less than $r_{S}$. 
- The seller will introduce into the auction, for every item $i$, a reserve bidder bidding the amount $r_{i}$ for the package $\{i\}$.

The main discrepancy here is that any payment (including the "payments" made by reserve bidders) must be greater than or equal to the package reserve amount. This forces the "payment" within the algorithm for any reserve bidder to be exactly equal to the reserve amount $r_{i}$, which in turn forces the seller to be fully compensated by a potential blocking coalition if it involves a reserve bidder. For example, applied to the previously considered scenario, we would still have $p_{1}=30$, but with a VCG payment for reserve bidder 4 under the reserve amount 10, we are forced to set the initial payment $p_{4}=10$. The bid by bidder 2 is no longer blocking, as she cannot overcome the additional 10 units that must be compensated to the seller to obtain item $B$, which appear as payments from the fictional bidder 4. Although logically equivalent, the two rules above were deemed more appealing than the following possibility based on the "reduced bid," which can be thought of as "moving the origin" according to the reserve vector, and then "moving it back" at the end of the auction:

- The seller will specify a reserve amount for each item, $r_{i}$, stipulating that each bid $b_{j}(S)$ be reduced by the amount $r_{S}$ prior to the winner determination and CCG implementation.

- Each bidder $j$ winning package $S_{j}$ will pay the amount $p_{j}$ determined using reduced bids, plus the base reserve amount $r_{S}$.

Relative to the equivalent former pair, this latter pair of rules seems a bit more confusing for participating bidders, because it introduces a second reduction of bids that is different from the "surplus-based" reduction of bids that occurs in the CCG computation of a core outcome. It might also be unclear at first glance that the "movement of the origin" in bid space prior to running the algorithm, followed by an equivalent "move-back" in payment space at the end of the auction is a non-trivial operation.

Next, we note a different reserve-setting procedure, appropriate for a seller who has zero value for keeping any item but wishes to set reserve payments in order to ensure adequate compensation is received when there is a lack of competition on a particular item or bundle. (For example, a spectrum authority might not have any value for holding a spectrum license unsold but also would like to charge a nominal fee.) This procedure is implemented by simply setting a lower bound $r_{S}$ on any bundle $S$, without the insertion of dummy bidders. In this case we see that if we compared a situation where an item $i$ went unsold and bidder $j$ is awarded set $S_{j}$ with bid amount $b_{j}\left(S_{j}\right)=a$, to an alternative solution in which bidder $j$ is awarded set $S_{j} \cup\{i\}$ with a bid of $b_{j}\left(S_{j} \cup\{i\}\right)=a$, there would be no change in social welfare, consistent with a seller who literally has zero value for keeping item $i$.

Next we note, via example, a peculiar finding regarding the use of dummy "reserve bidders" (as previously outlined) and the more simple use of "bounds-only" reserves on any bundle payment (as in the previous paragraph). Consider the following example.

EXAMPLE 5.

\begin{tabular}{llcc}
\hline & Bids & $\begin{array}{c}\text { Reserve } \\
\text { bidders }\end{array}$ & $\begin{array}{c}\text { Bounds } \\
\text { only }\end{array}$ \\
\hline$b_{1}(A B)=100$ & $b_{2}(C D)=100$ & $p_{1}=55$ & $p_{1}=45$ \\
$b_{3}(B C)=90$ & $\begin{array}{c}\text { Seller reserve for } \\
\text { each item, } r_{i}=10\end{array}$ & $p_{2}=55$ & $p_{2}=45$ \\
& & \\
\hline
\end{tabular}

If the seller employs reserve bidders (and bounds as outlined above) or equivalently runs the auction after reducing bids by reserve amounts and adds the reserve amounts back into final payments, the result is higher payments than if the seller simply set a lower bound on any bundle payment of $10|S|$. This result seems intuitive; if the seller is aggressively bidding for items, this might drive up the prices on those items. But the result is reversed in the following example.

EXAMPLE 6.

\begin{tabular}{lccc}
\hline & Bids & $\begin{array}{c}\text { Reserve } \\
\text { bidders }\end{array}$ & $\begin{array}{c}\text { Bounds } \\
\text { only }\end{array}$ \\
\hline$b_{1}(A)=100$ & $b_{2}(B)=100$ & $p_{1}=35$ & $p_{1}=45$ \\
$b_{3}(A B C D)=90$ & $\begin{array}{c}\text { Seller reserve for } \\
\text { each item, } r_{i}=10\end{array}$ & $p_{2}=35$ & $p_{2}=45$ \\
& & \\
\hline
\end{tabular}

In both Examples 5 and 6, the reserve bounds are always loose for the actual winners. For Example 5, we see that the dummy bidders participate in coalition formation but are not part of the efficient solution; losing bidder 3 forces the winning bidders to pay at least 90 on her own, but at least 110 with the help of reserve bidders who are willing to buy back the items $A$ and $D$ at 10 each.

In Example 6, we see the reverse phenomenon; the active reserve bidders participate in the efficient solution but not in coalition formation for price setting. Without reserve bidders, the two winners need to raise 90 units of revenue to ensure that bidder 3 is not blocking. When the reserve bidders are present, however, the winning bidders "get help" from the reserve bidders, who contribute 20 to buy back the items $C$ and $D$, and thus 20 less is collected from the actual winning bidders. Note that depending on the actual utility function of the seller, both outcomes are logically consistent. If the seller actually perceives a loss of 10 units of value for giving away an item, then for Example 6, the seller perceives 90 units of utility from her revenue combined with her value for keeping items $C$ and $D$. If the seller instead had no value for the items she keeps, then she gets 90 units of utility from revenue only.

Thus these two approaches to reserve setting are highly dependent on the utility of the seller for keeping items, and the revenue implications of choosing one method over the other are not always clear. So for a telecommunications authority like Ofcom, we recommended "bounds 
only" reserve setting because they did favor allocating as much spectrum as possible. But for the FAA we recommended "reserve bidders" because the potential reduction of delay from unallocated slots had value according to the FAA's objectives, who planned to (potentially) retire slots that were not sold at auction.

We note finally that the reserve bidder technique, since equivalent to a reduction of each bundle bid by the bundle reserve amount, does indeed represent a "shift of the origin" in payment space, and thus like a constant- $p^{0}$ reference results in payments that are highly sensitive to changes in the reserve structure set by the auctioneer before the auction. For example, a one-for-one shift of the reserve amounts so that $r_{A}=11$ and $r_{B}=9$ in Examples 5 and 6 results in the identical shift in payments by the bidders when using a reserve-bidder scheme. Final payments become $(56,54)$ and $(36,34)$ in Examples 5 and 6 , respectively. But with a bounds-only technique, payments are relatively insensitive to changes in the seller-specified reserve amount. In general, unless a bidder is paying an amount exactly equal to the seller-specified bundle reserve amount, the choices made by the auctioneer regarding bundle reservation value are inconsequential under a boundsonly method, while the previous argument showed that this is not true of a reserve-bidder approach.

\section{Applications}

The practical applicability of the techniques proposed here is limited mainly by our computational ability to solve larger and more complex winner determination problems. For assurance of a timely auction in real life, we would usually like to guarantee worst-case run times for any winner determination problem within a few minutes or hours. In practice, computational run time was not an issue at all during our testing for Ofcom, in which we reviewed several hundred test cases in anticipation of the United Kingdom's three spectrum auctions. In all testing, we implemented the algorithm described in $\$ 3.2$ using CPLEX 11.1, and test cases were run in parallel by consulting company dotEcon and by associates at the Smith Institute for Industrial Mathematics and System Engineering. Run times were consistently under 20 minutes for even the worst cases, with the median cases taking a few seconds or less to solve. For the two real-world auctions, run times for winner and payment determination were around 1 second.

A few other practical points regarding the applications of the techniques proposed here are outlined in Appendix A.6. One minor point of caution did arise in our testing, which we mention here, however. In the assignment stage of the $10-40 \mathrm{GHz}$ or of the $2.6-\mathrm{GHz}$ auctions, Ofcom wanted to assure that any unsold spectrum was kept as one contiguous block so that it could be readily used or resold at a later date. This condition was solved easily enough with an IP formulation by having an appropriately sized space-holding bid for unsold blocks, and having the IP determine a partition of the spectra within a category because the quantities of lots had already been determined in the principal stage. But care must be taken with a partitioning formulation when computing either the VCG payments or the core payments.

Specifically, if we tried to find a VCG payment by removing a bidder, the partitioning IP became infeasible. The simple solution is to not remove a bidder, but instead lower all of his bids to zero for his VCG computation. Similarly, when attempting to separate a violated core constraint, we reduce each bid by the bidder's current surplus. When solving the ensuing partitioning formulation IP, we noticed that it was necessary to replace any negative bid with zero, or else certain blocking coalitions would be ignored. Furthermore, the algorithm as proposed in $\$ 3.2$ said that a constraint was generated with a 1 for every bidder not receiving items in the separation IP solution, but every bidder must receive items in the assignment stage partitioning formulation. Again, the simple fix is to place a 1 into the constraint for any bidder who is forced to take a zero-valued bundle (under the surplus adjusted bids). These are fairly straightforward modifications of the coreselection algorithm, but we include these facts for completeness that a slightly different procedure must be taken in winner-determination problems for which each bidder must receive items.

\section{Computational Experiments}

In this section we describe the results of a set of computational experiments performed using data generated by the Combinatorial Auction Test Suite (CATS) as introduced by Leyton-Brown et al. (2000). The CATS software simulates bidding behavior in a number of realistic economic environments, for example, when bidders are interested only in bundles of contiguous geographic regions in a spectrum license auction, or in bundles that form a path in a shipping-lane auction, etc. We used the same instances used by Day and Raghavan (2007) (which are available at http://users.business.uconn.edu/bday/CATSCCG.zip), restricting to the auctions for 16,32 , and 64 items. Among these instances with three different sizes for the number of auction items, we allowed the CATS number-of-bids parameter to vary among the values $\{10,25,50,100,250,1,000\}$, and we replicated each of these parameter values 50 times, for a total of 1,050 randomly generated auction instances.

All instances were run using CPLEX 11.1 on a Windows Vista, AMD Turion $642-\mathrm{GHz}$ processor with $2 \mathrm{~GB}$ RAM. Relative to the earlier computations performed by Day and Raghavan (2007) on these instances, all worstcase and average run times (with one exception) actually decreased; the computational gains from an upgrade to CPLEX 11.1 from CPLEX 9.0 more than outweighed the increased burden of solving quadratic programs to select among MRC-points, and the increased burden of 
the randomized tie-breaking rule put in place for the FAA slot auction, which itself constituted an additional winnerdetermination run. The only exception was in the worstcase run time for 64 items and 1,000 bids, which increased from 3,703 to 7,583 seconds, but this entire increase could be accounted for by the additional winner determination needed to implement the randomized tie-breaking rule $(6,472$ seconds were spent in the winner-determination phase, which included both runs). When this single instance is removed, all worst-case run times are an improvement over the Day and Raghavan (2007) results. All 16-item auctions concluded in under 2.5 seconds, all 32-item auctions in under 30 seconds, and all 64-item auctions in under 2.5 hours (under 49 minutes when the single worst case was removed). The average performances for the largest 1,000 bid cases were $0.96,5.6$, and 611 seconds, for 16,32 , and 64 items, respectively, indicating that this class of algorithms does indeed perform in a comfortable time-scale for these auction sizes.

Also as in Day and Raghavan (2007), we found that a large minority (roughly 42\%) of these CATS instances result in VCG outcomes when a VCG-nearest rule is applied. Considering all instances in this study, the VCG outcome delivers an average of $42 \%$ surplus to the bidders, while the VCG-nearest MRC rule delivers $33 \%$ surplus, leaving about $9 \%$ of value as a potential benefit from unilateral misrepresentation of preferences, because the VCG payment gives the maximum amount of benefit available from a unilateral deviation. Restricting only to instances in which the VCG outcome differs from an MRC outcome (about 58\% of instances), the average bidder surplus becomes $33 \%$ for VCG, $18 \%$ for the VCG-nearest MRC rule, leaving a maximum of about $15 \%$ of value available through unilateral misrepresentation. Thus the majority of the possible benefits of bid shading are removed by this quadratic core-selecting rule, relative to a pay-as-bid rule. These results are summarized in Table 1. Also, although $15 \%$ of value might seem substantial, one should remember that this measures the maximum possible gain from deviation, assuming that the bidder knows to shade by this amount and not more, and that opponents do not shade their bids as well.

\subsection{VCG-Nearest vs. Zero-Nearest}

With Example 3 on page 596, we showed that the use of a zero-nearest reference rule (i.e., when $p^{0}=\overrightarrow{0}$ ) can result in a high-valued winner shouldering little if any of the monetary burden of overcoming a blocking coalition. Here we show that this phenomenon is not peculiar to a carefully

Table 1. Average bidder surplus as a $\%$ of value.

\begin{tabular}{lcc}
\hline Method & All instances $(\%)$ & MRC $\neq$ VCG instances $(\%)$ \\
\hline VCG & 42 & 33 \\
MRC & 33 & 18 \\
\hline
\end{tabular}

constructed example, but instead that it occurs frequently when using a random data-set, in this case the CATS data. Toward this end, we duplicated the runs described above using a zero-nearest MRC reference rule rather than a VCG-nearest MRC rule. Then we looked at the difference between the final MRC payment and the VCG payment for each bidder. For any instance in which this amount was positive, and for which there were at least two winners, we isolated the highest-valued and lowest-valued winning bidder and measured the percentage of increase from the VCG total that was paid for by each of these two bidders. That is, we computed

$\frac{p_{\bar{j}}^{*}-p_{\bar{j}}^{\mathrm{VCG}}}{\sum_{j \in N}\left(p_{j}^{*}-p_{j}^{\mathrm{VCG}}\right)}$,

where $\bar{j}$ was the index of the highest-valued winning bidder, and the lowest-valued winning bidder, respectively. Using this measure, we confirmed that the intuition shown by Example 3 did indeed persist. (In that example, this statistic took the values 0 and 1 , for the highest- and lowestvalued winners, respectively, under the zero-nearest rule, and $(0.5,0.5)$ under the VCG-nearest rule.) For the VCGnearest computations, this statistic (15) averaged roughly $20 \%$ for the highest-valued winner, while the zero-nearest computations resulted in a value of about $6 \%$ for the highest-valued winner; the use of a zero-nearest rule results in high-valued winners shouldering less of the burden of overcoming blocking coalitions when the VCG outcome is not in the core. Similarly, we found that the lowest-valued winner paid about $7 \%$ of the burden under a VCG-nearest rule, while they paid $12 \%$ under the zero-nearest implementation. Furthermore, the extreme behavior of Example 3 , in which the higher-valued winner paid none of the burden of overcoming a blocking coalition (beyond the VCG payment) was also observed in the CATS data. This phenomenon, in which the statistic (15) equalled zero for the highest-valued winning bidder, occurred in only $8 \%$ of the relevant instances under a VCG-nearest rule, but over $32 \%$ of the instances using a zero-nearest rule showed this extreme lopsided-ness. Figure 2 visually indicates the overall disproportionate burden placed on the lowest-valued winner relative to the highest-valued winner under a zeronearest rule. Clearly, from viewing these graphs, this disparity between the two approaches is most pronounced when the number of winners is small. (Using the CATS data, the presence of more bids makes it more likely to have more winners.)

\subsection{Reserve Bidders vs. Bounds-Only Reserves}

To observe the effect of the two seller-reserve formats discussed here, we ran each CATS auction instance using a VCG-nearest MRC reference rule, once using the boundsonly approach and once using the reserve-bidders approach. Although Examples 5 and 6 demonstrated that drastically 
Figure 2. When total payments are more than the total VCG payments, these graphs show the proportion of the difference paid (on average) by the winner with the highest valued bundle, the lowest valued bundle and all other winners, under (a) the VCG-nearest rule, and (b) the zero-nearest rule, as a function of the number of bids parameter.

(a) VCG-nearest

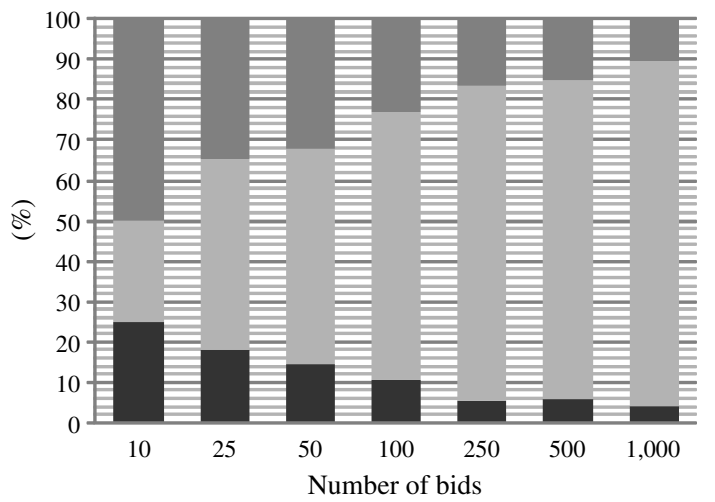

different outcomes could occur in some specially constructed examples and that neither was a universally better approach, we wanted to demonstrate that the discrepancies between the two approaches persisted in the robust environment provided by the CATS data. Because the CATS data did not generate a seller-reserve value for each item, we arbitrarily asserted a reserve value of $r_{i}=20$ monetary units for half of the items in each auction instance, and reserve value $r_{i}=40$ for the other half of the items. After this we pruned out any bids that did not meet the implied (additive) bundle reserve value, and we removed any auction instances for which fewer than two bids remained, leaving us with 706 or roughly $67 \%$ of the original auction instances for further investigation.

As expected, a bounds-only approach tended to cause an increase in the number of units sold, but only a modest increase; for these data, less than $66 \%$ of items were sold if reserve bidders were used, and just above $70 \%$ were sold under the bounds-only approach. If considering a seller who has no value for keeping an item, and thus whose utility is specified exactly by the amount of revenue generated, we found that about $56 \%$ of the instances experienced an increase in revenue under the bounds-only approach, although the overall performance showed a small (1\%) average reduction in revenue with the bounds-only approach. In the extreme cases, the largest positive effect of switching to bounds-only was a $78 \%$ increase in revenue, while the largest negative effect was a nearly $40 \%$ reduction in revenue with bounds-only. But mostly, the revenue effects were noticeable but modest, averaging a $6 \%$ absolute deviation between the two reserve formats. These results indicate that in specific circumstances, the effect of reserve-format selection might be substantial in either direction, but that these large discrepancies are not typical (79\% of the instance has an absolute revenue change of $10 \%$ or less from switching approaches). Also as might be expected, the revenue effect of the reserve-format selection had less impact on auctions with a larger number of bids. When auctions with fewer than 250 bids were removed (b) Zero-nearest

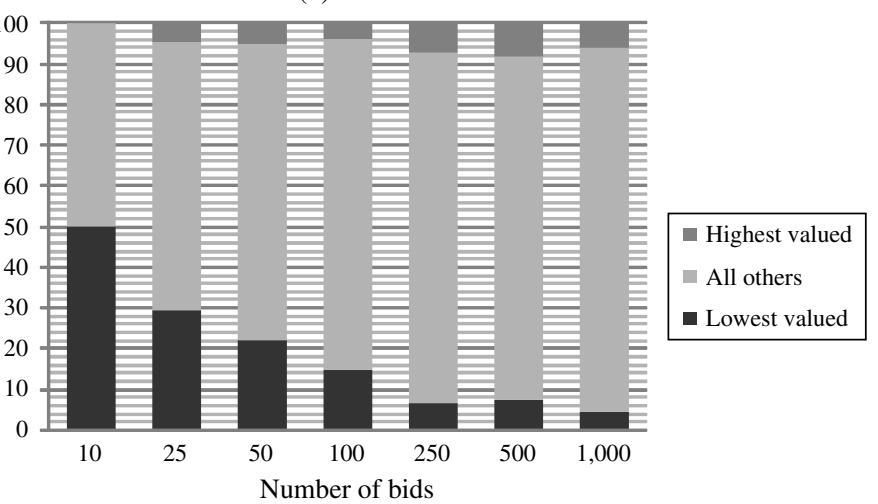

from our analysis, 99.6\% had a revenue change of less than $10 \%$ when switching approaches, and here we saw a $1 \%$ average increase in revenue over all instances when switching to bounds-only. Also, when these auctions with a small number of bids were removed, the extreme cases were much less extreme; revenue decreased by no more than $7 \%$, and increased by no more than $11 \%$ when switching to bounds-only.

The full set of total revenue comparisons is given in Figure 3, where each vertical bar represents one of the 706 instances, grouped by the number of items in the auction $(16,32$, or 64$)$ and ordered within a group by the number of bids as indicated on the horizontal axis. Values on the vertical axis indicate total auction revenue as a percentage of the revenue sum of the two methods for that instance; thus $50 \%$ indicates equal revenue across the two methods, $66 . \overline{6} \%$ indicates that one method had twice the revenue of the other, etc. This picture indicates that the direct revenue comparison is indeed unclear but seems visually to slightly favor the reserve-bidder approach if just considering revenue, consistent with the overall $1 \%$ revenue disadvantage of bounds-only indicated above. Also, we see that regardless of the number of items being sold, the discrepancy between the two approaches becomes less pronounced with more bids in the auction, indicated by the convergence to the $50 \%$ line as we move from left to right in each itemquantity group.

When we consider a seller who does value keeping unsold items, the seller's net utility from the auction is then the value of the revenue received minus the value of the items sold. In this case our results more clearly favor a reserve-bidder format for this type of seller, although again, not in all instances. In about $7 \%$ of these instances (including auctions with both many and few bids) a seller would relinquish $100 \%$ of her utility if choosing a boundsonly approach over a reserve-bidder approach. Although a single extreme instance did show a nearly $105 \%$ increase in seller utility under bounds-only, $71 \%$ of all instances showed a reduction of seller utility when switching to 
Figure 3. Revenue comparison of the reserve bidder and bounds-only approaches over all relevant instances.

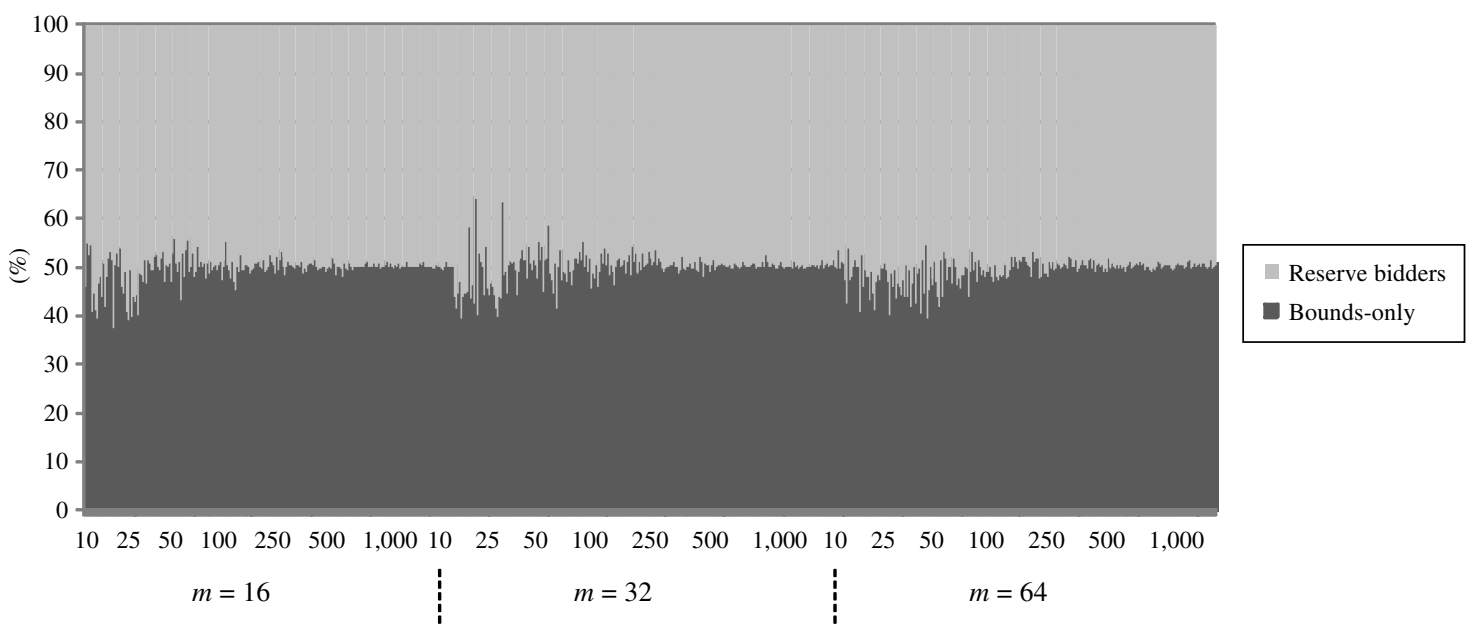

bounds-only. Furthermore, of the roughly $29 \%$ showing an increase in seller utility, 95\% showed only a modest increase of less than $15 \%$. On average, switching to a bounds-only approach resulted in a $15 \%$ loss of seller utility. This average effect was much less for auctions with less than 250 bids, only a $1.5 \%$ reduction in average sellerutility when switching to bounds-only. Furthermore, the scope for large increases in seller utility in extreme cases nearly disappeared when looking only at these many-bid instances; the biggest increase when switching to boundsonly then became about $9 \%$.

\section{Conclusions}

We presented a general algorithm for selecting among core outcomes for use in any combinatorial auction, and we described the many beneficial properties of the approach. We motivated the idea that quadratic core-pricing is simple to understand (via Example 1) but that it is also general enough and extensible enough to handle the full complexity of any combinatorial auction problem. For instances in which the winner-determination problem can be solved in a reasonable time, then "fair" payments can also be computed in a reasonable time. This development represents an important milestone in operations research, in which a computational, algorithmic development opens the door to efficient solutions for a wide class of economic resourceallocation problems.

The prices we generate represent a natural generalization of the second-price paradigm from single-item auctions. Among core-selecting mechanisms, the pricing rule minimizes the incentives for bidders as a whole to misreport their true values for packages. Using standard nonlinear programming tools, we demonstrated how the underlying mathematics induces an equitable decomposition of payments, so that different bidders each contribute an equal amount for any payment goal that they must achieve as a group. We also showed the computational simplicity of the specific quadratic programming problem we encounter, and we demonstrated several different interpretations and formulations of the core, each one providing a different perspective to help motivate the core property as a natural requirement in combinatorial auctions.

Our computational experiments lent further support for the practical viability of these computational techniques, using a standard benchmark from the CA literature. These experiments also helped to demonstrate some of the seeming distortions that arise when a zero-nearest version of the algorithm is implemented, in which the proportion of the burden needed to overcome a blocking coalition is unevenly spread across winners. Because any constant reference rule is equivalent to a zero-nearest reference rule with the corresponding change of coordinates, this motivates our slight preference for VCG-nearest rules in practice. As the concurrent work of Erdil and Klemperer (2009) begins to hint, though, there might be alternative dynamic selection rules, which are, like the VCG-nearest rule, not skewed by pre-auction parameter settings made by the auctioneer, but this remains the subject of future study. Also, our computations indicated that the selection of a reserve scheme might be less trivial than it first appears, and this as well remains a interesting avenue of future research.

As the benefits of this class of quadratic payment determination algorithms become more well-known, we expect further applications to emerge. Based on preliminary presentations of this research and the early successes of the auctions held in the United Kingdom, the FAA adopted this pricing rule for the auction of landing-slot rights in the three New York City airports. To minimize disruption to the status quo, the plan was to auction only a small portion of time-slots at the three airports. As a result, the associated winner-determination problems solve easily using standard, off-the-shelf-software such as CPLEX. The greatest challenge for the landing-slot application, however, has proved to be the politics of auctioning, not any difficulties in computing winners or payments. 


\section{Electronic Companion}

An electronic companion to this paper is available as part of the online version at http://dx.doi.org/10.1287/opre.1110.1024.

\section{Endnotes}

1. In December 2008, one month prior to the actual auction of landing rights for the three New York City airports, a federal court stayed the auction. The plans for auctioning slots are now uncertain.

2. Of course, we multiply the objective by $\frac{1}{2}$ prior to taking a gradient, as is typical in quadratic optimization.

3. Note that this is always true for the VCG-nearest rule, while other selections of $p^{0}$ would require additional arguments to justisfy $p^{0}$ as a lower bound on payments.

4. We thank Larry Ausubel for helping to devise this simple example.

\section{References}

Ausubel, L., P. Cramton. 2003. Vickrey auctions with reserve pricing. Econom. Theory 23(3) 493-505.

Ausubel, L., P. Milgrom. 2002. Ascending auctions with package bidding. Frontiers Theoret. Econom. 1(1) 1-42.

Ausubel, L., P. Milgrom. 2006. The lovely but lonely Vickrey auction P. Cramton, Y. Shoham, R. Steinberg, eds. Combinatorial Auctions, Chap. 1. MIT Press, Cambridge, MA, 17-40.

Ausubel, L., P. Cramton, P. Milgrom. 2006. The clock-proxy auction: A practical combinatorial auction design. P. Cramton, Y. Shoham, R. Steinberg, eds. Combinatorial Auctions, Chap. 5. MIT Press, Cambridge, MA, 115-138.

Bazaraa, M., H. Sherali, C. Shetty. 1979. Nonlinear Programming: Theory and Algorithms. Wiley and Sons, New York.

Bernheim, B., M. Whinston. 1986. Menu auctions, resource allocation and economic influence. Quart. J. Econom. 101(1) 1-31.

Bichler, M., P. Shabalin, A. Pikovsky. 2009. A computational analysis of linear price iterative combinatorial auction formats. Inform. Systems Res. 20(1) 33-59.

Cramton, P. 2006. Simultaneous ascending auctions. P. Cramton, Y. Shoham, R. Steinberg, eds. Combinatorial Auctions, Chap. 4. MIT Press, Cambridge, MA, 99-114.

Cramton, P. 2009. Spectrum auction design. Working paper, University of Maryland, College Park.

Cramton, P., Y. Shoham, R. Steinberg, eds. 2006. Combinatorial Auctions MIT Press, Cambridge, MA.

Day, R., P. Milgrom. 2008. Core-selecting package auctions. Internat. J. Game Theory 36(3) 393-407.

Day, R., S. Raghavan. 2007. Fair payments for efficient allocations in public sector combinatorial auctions. Management Sci. 53(9) 1389-1406.

deVries, S., R. Vohra. 2003. Combinatorial auctions: A survey. INFORMS J. Comput. 15(3) 284-309.

Erdil, A., P. Klemperer. 2010. A new payment rule for core-selecting package auctions. J. Eur. Econom. Assoc. 8(2-3) 537-547.
Goldberg, A., J. Hartline. 2003. Envy-free auctions for digital goods. $A C M$ Conf. Electronic Commerce (EC '03), Association for Computing Machinery, Inc., San Diego, June 2003.

Krishna, V. 2002. Auction Theory. Academic Press, Boston.

Lamy, L. 2010. Core-selecting package auctions: A comment on revenuemonotonicity. Internat. J. Game Theory 39(3) 503-510.

Leyton-Brown, K., M. Pearson, Y. Shoham. 2000. Towards a universal test suite for combinatorial auction algorithms. EC 'OO Proc. 2nd ACM Conf. Electronic Commerce. ACM Press, New York, 66-76.

Milgrom, P. 2004. Putting Auction Theory to Work. Cambridge University Press, Cambridge, UK.

Myerson, R. B. 1981. Optimal auction design. Math. Oper. Res. 6(1) $58-73$.

Nisan, N. 2006. Bidding languages in combinatorial auctions. P. Cramton, Y. Shoham, R. Steinberg, eds. Combinatorial Auctions, Chap. 9. MIT Press, Cambridge, MA, 215-232.

Othman, A., T. Sandholm. 2010. Envy quotes and the iterated coreselecting combinatorial auction. Proc. Natl. Conf. Artificial Intelligence (AAAI)

Ott, M. 2009. Second-price proxy auctions in bidder-seller networks. Doctoral dissertation, Karlsruhe Institute of Technology, Karlsruhe, Germany.

Parkes, D., J. Kalagnanam, M. Eso. 2001. Achieving budget-balance with Vickrey-based payment schemes in exchanges. Proc. 17th Internat. Joint Conf. Artificial Intelligence (IJCAI'01), 1161-1168.

Porter, D., S. Rassenti, A. Roopnarine, V. Smith. 2003. Combinatorial auction design. Proc. Natl. Acad. Sci. USA 100(19) 11153-11157.

Rassenti, S., V. Smith, R. Bulfin. 1982. A combinatorial auction mechanism for airport time slot allocation. The Bell J. Econom. 13(2) $402-417$

Rothkopf, M. 2007. Thirteen reasons why the Vickrey-Clarke-Groves mechanism is not practical. Oper. Res. 55(2) 191-197.

Rothkopf, M., A. Pekeč, R. Harstad. 1998. Computationally manageable combinatorial auctions. Management Sci. 44(1) 131-1147.

Rothkopf, M., T. Teisberg, E. Kahn. 1990. Why are Vickrey auctions rare? J. Political Econom. 98(1) 94-109.

Robert W. Day is an associate professor of operations and information management in the School of Business at the University of Connecticut. His work on combinatorial auctions (i.e., auctions with package bidding) received the Dantzig dissertation award in 2005 and the INFORMS Computing Society Prize in 2008. His research interests also include optimization-based methods in hospital management.

Peter Cramton is professor of economics at the University of Maryland. Since 1983, he has conducted widely cited research on auction theory and practice. The main focus is the design of auctions for many related items. Applications include auctions for radio spectrum, electricity, financial securities, diamonds, and timber. He has introduced innovative market designs in many industries. 


\section{e - c o m p a n i o n}

Electronic Companion-“Quadratic Core-Selecting Payment Rules for Combinatorial Auctions" by Robert W. Day and Peter Cramton, Operations Research, http://dx.doi.org/10.1287/opre.1110.1024. 


\section{Online Appendix to "Quadratic Core-Selecting Payment Rules for Combinatorial Auctions," by Day and Cramton}

\section{A.1 Semi-sincere strategies eliminate incremental envy}

To further motivate why a bidder would choose to implement a semi-sincere strategy (since there may exist other best-response strategies) we note the following:

Definition 7.1 Given an auction outcome, a bidder is incrementally envy-free if she would not prefer to additionally buy any collection of her opponents' awarded bundles at the respective prices they pay.

Lemma 7.2 If a winning bidder employs a semi-sincere strategy in any core-selecting auction, then she is incrementally envy-free.

Proof. Without loss of generality, suppose that each bidder $j$ wins bundle $S_{j}$, and that bidder 1 incrementally-envies the bundles of bidders $2,3, \ldots \mathrm{k}$. That is, bidder 1 who is awarded the bundle $S_{1}$ and pays $p_{1}$, would prefer to receive the bundle $S_{1} \cup S_{2} \cup \ldots S_{k}$ and pay $p_{1}+p_{2}+\ldots p_{k}$. Letting $v_{j}(S)$ represent bidder $j$ 's value for a bundle $S$, this means that:

$$
\begin{aligned}
& v_{1}\left(S_{1} \cup S_{2} \cup \ldots S_{k}\right)-\sum_{j=1}^{k} p_{j}>v_{1}\left(S_{1}\right)-p_{1} \\
& b_{1}\left(S_{1} \cup S_{2} \cup \ldots S_{k}\right)-b_{1}\left(S_{1}\right)>\sum_{j=2}^{k} p_{j}
\end{aligned}
$$

with (17) following from (16) by the definition of the semi-sincere strategy. But employing a constraint of the form (6) as defined on page 13, where $W$ represents the set of all bidders receiving non-empty bundles, we have:

$$
\begin{array}{r}
\sum_{j=2}^{k} p_{j} \geq w d(N \backslash\{2,3, \ldots k\})-\sum_{j \in W \backslash\{2,3, \ldots k\}} b_{j}\left(S_{j}\right) \\
b_{1}\left(S_{1} \cup S_{2} \cup \ldots S_{k}\right)-b_{1}\left(S_{1}\right)>w d(N \backslash\{2,3, \ldots k\})-\sum_{j \in W \backslash\{2,3, \ldots k\}} b_{j}\left(S_{j}\right) \\
b_{1}\left(S_{1} \cup S_{2} \cup \ldots S_{k}\right)+\sum_{j \in W \backslash\{1,2,3, \ldots k\}} b_{j}\left(S_{j}\right)>w d(N \backslash\{2,3, \ldots k\})
\end{array}
$$

where (18) is simply the core constraint of the form (6); (19) follows transitively from (17), and (20) following after canceling terms. But (20) is a contradiction; a collection of disjoint bundle bids made by a subset of the players in $N \backslash\{2,3, \ldots k\}$ is greater than $w d(N \backslash\{2,3, \ldots k\})$, which is defined as maximal over such combinations 
of bids.

This lemma motivates not only that a bidder can find a utility-maximizing strategy among her possible semi-sincere strategies, but also that she will not envy any collection of her opponents' bundles, a property not captured in her quasi-linear utility function. We also note that the existence of a best reply in semisincere strategies does not imply incremental-envy-freeness, as the VCG auction always has a best reply in semi-sincere strategies (with $\alpha_{j}=0$ for all bidders) but may not satisfy this property when outcomes are not in the core.

\section{A.2 Efficacy of Core Constraint Generation}

Next we provide a few details that are of theoretical interest, concerning the efficacy of this CCG approach. One natural question to ask is whether the constraints generated ever end up to be redundant, or loose constraints in the final solution. For example, is it ever the case that at iteration $t$ we find violated constraint $p a_{C_{t}} \geq \beta_{C_{t}}$, but later find, at termination, that $p^{*} a_{C_{t}}>\beta_{C_{t}}$ ? So far in our experiments, under a mild additional condition of minimality of $a_{C_{t}}$ at each iteration, the answer is no. To illustrate this minimality condition consider the following example, which is a slight modification of the main example from Day and Raghavan (2007), under a VCG-nearest MRC-reference rule. Here, $m=3$, the item-set is $\{A, B, C\}$, and $n=9$ (single-minded) bidders have the following bids:

$$
\begin{array}{rlcc}
b_{1}(A)=20 & b_{2}(B)=20 & b_{3}(C)=20 \\
\text { Example 2: } & b_{4}(A B)=28 & b_{5}(A C)=26 & b_{6}(B C)=23 \\
b_{7}(A)=10 & b_{8}(B)=10 & b_{9}(C)=10
\end{array}
$$

The unique efficient allocation is to accept the single bids of bidders 1,2 , and 3 , with $p^{0}=(10,10,10)$. Reducing each bid by current surplus, we find two candidates for $C_{1}$; both $\{4,7\}$ and $\{3,4\}$ would be willing to offer the seller 38 at the current prices, but the first selection results in $a_{C_{1}}=(1,1,1)^{T}$, $\beta_{C_{1}}=38$, while the other results in $a_{C_{1}}=(1,1,0)^{T}, \beta_{C_{1}}=28$. If we follow the algorithm through to termination using either selection, we do always arrive at the (unique) solution $p^{*}=(15.5,12.5,10.5)$. But if we generate the first of these possible constraints $p_{1}+p_{2}+p_{3} \geq 38$, we see that in the final solution this constraint becomes loose: $p_{1}^{*}+p_{2}^{*}+p_{3}^{*}=38.5>38$. The latter selection for $C_{1}$, on the other hand, has $(1,1,0)^{T}<(1,1,1)^{T}$, thus satisfying minimality among maximally violated coalitions at $p^{0}$, and indeed remains tight at termination; $p_{1}^{*}+p_{2}^{*}=28$. Also, one may note for this example that the use of a VCG-nearest reference rule without explicit payment minimization results in payments $(14 . \overline{6}, 13 . \overline{3}, 11 . \overline{3})$, which has a greater sum 39. $\overline{3}>38.5$, verifying that the explicit minimization of payments is necessary, if desired, and does not follow in general 
from the minimization of distance to a reference point, even though Example 1 and other small examples seem to suggest the reverse.

\section{A.3 Proof of Theorem 1}

Proof. Suppose for example (WOLOG) that winning bidder 1 uniformly increases her semi-sincere bids, i.e., if she originally submitted $b_{1}(S)=\max \left(0, u_{1}(S)-\alpha_{1}\right)$ for all bundles $S$, now she submits $\bar{b}_{1}(S)=$ $\max \left(0, u_{j}(S)-\alpha_{j}+\delta\right)$. First, we note that such an increase could not change the efficient ${ }^{5}$ solution, as the efficiency of any allocation involving bidder 1 increases by the same amount, and those not involving her do not change.

Now consider the various cases for each coalitional constraint in the core-selection program, $\sum_{j \in W \backslash C} p_{j} \geq$ $w d(C)-\sum_{j \in C} b_{j}\left(S_{j}\right)$. If Bidder 1 does not belong to coalition $C$, clearly this constraint remains unchanged. Further, if bidder 1 does belong to $C$, and bidder 1 would receive items in the winner-determination problem solved over $C$, then the terms $w d(C)$ and $b_{1}\left(S_{1}\right)$ would both increase by $\delta$, resulting in a cancellation, and the constraint again remains unchanged.

Finally, if bidder 1 belongs to $C$ but does not receive items under $w d(C)$, the $C$-indexed constraint will be relaxed by $\delta$ when bidder 1 switches from bids $b$ to $\bar{b}$, but this relaxation is inconsequential since the constraint must already be loose at optimality, and the relaxation of loose constraints will not affect the optimization. To see that the $C$-indexed constraint is indeed loose at $p^{*}$ in this case, note that since bidder 1 receives no goods in the solution to $w d(C)$, we have $w d(C)=w d(C \backslash\{1\})$. By the feasibility of $p^{*}$ we have $\sum_{j \in W \backslash(C \backslash\{1\})} p_{j}^{*} \geq w d(C \backslash\{1\})-\sum_{j \in(C \backslash\{1\})} b_{j}\left(S_{j}\right)$, and then subtracting $p_{1}^{*}<b_{1}\left(S_{1}\right)$ which holds by assumption, we recover $\sum_{j \in W \backslash C} p_{j}^{*}>w d(C)-\sum_{j \in C} b_{j}\left(S_{j}\right)$, as desired. Since individual rationality constraints were also loose at optimality, we have shown that all constraints defining the core either remain the same or are the relaxation of already loose constraints at optimality. Together with the assumption that the reference vector $p^{0}$ has not changed, this results in no change to $p^{*}$ following the increase in semi-sincere strategy.

\section{A.4 Proof of Lemma 3.1}

Proof. If a constraint is necessary, then there will be some $\epsilon>0$, for which the constraint is still tight when relaxed by $\epsilon$, and for which the solution must change. Suppose that there is a $j \in N$, such that $p_{j} \leq b_{j}\left(S_{j}\right)$ is a necessary constraint. A relaxation of this constraint by $\epsilon$ corresponds to an increase of $b_{j}\left(S_{j}\right)$ by $\epsilon$,

\footnotetext{
${ }^{5}$ This assumes the use of a tie-breaking rule that is robust against such an increase. In the U. K. spectrum auctions, for example, each item in each bundle bid received a randomly generated number, and the sum became the objective function in a secondary WD optimization, constrained to be efficient.
} 
which leaves the efficient solution unchanged. But now for a VCG-nearest rule, the KKT necessary and sufficient conditions form the same linear system, since the only affected condition is that of bidder $j$, i.e., $p_{j}^{*}=p_{j}^{0}+\sum_{a_{C} \in \tilde{A} \mid j \notin C} z_{C}-v-w_{j}$, which remains unchanged when the bid on $b_{j}\left(S_{j}\right)$ increase by $\epsilon$. This is because $p_{j}^{0}=b_{j}\left(S_{j}\right)-w d(N)+w d(N \backslash j)$, and the increase by $\epsilon$ in $b_{j}\left(S_{j}\right)$ is canceled by the increase in $\epsilon$ of $w d(N)$. But now the same linear system will have the same solution when the constraint is relaxed by $\epsilon$, and the constraint was not necessary. Since the constraint can then be removed, the result follows.

An illustration of this point can be observed in a similar perturbation of Example 1 to the one on page 16. Suppose that bidder 1 bid $b_{1}(A)=16$, and hold the remainder of the example unchanged. Now, compare the use of the VCG-nearest rule, and the constant-reference rule with $p^{0}=(14,12)$. Under the VCG nearest rule, the IR constraint $p_{1} \leq 16$, is loose at the optimal point $(15,17)$, as the VCG point responds to bidder 1's lower bid, increasing the VCG payment of bidder 2. But the constant-reference rule with $p^{0}=(14,12)$ results in final payments $(16,16)$, and without the IR constraint $p_{1} \leq 16$, would result in the non-IR point $(17,15)$. In neither case is the MRC constraint necessary, so the former case has payment decomposition $p_{1}^{*}=14+1, p_{2}^{*}=16+1$, while the latter case requires a $w_{1}$ term: $p_{1}^{*}=14+4-2, p_{2}^{*}=12+4$.

\section{A.5 Nonuniqueness of a payment decomposition and solving (QP) iteratively as constraints are generated}

If the constraint qualification condition arises that the columns of $\tilde{A}$ together with the 1-vector are linearly independent, then the $(v, w, z)$ combination (guaranteed to exist by KKT) is unique. But even in a simple example, such as Example 2, this constraint qualification may fail and the payment decomposition will not be unique.

For Example 2, we find that:

$$
\tilde{A}=\left[\begin{array}{lll}
1 & 1 & 0 \\
1 & 0 & 1 \\
0 & 1 & 1
\end{array}\right]
$$

with $p^{0}=(10,10,10), p^{*}=(15.5,12.5,10.5)$, and an infinite number of $(v, z)$ satisfying (KKT), including, for example, $\left(v^{1}, z^{1}\right)=(-4.5,6,4,1)$ and $\left(v^{2}, z^{2}\right)=(-3.5,5.5,3.5,0.5)$. In this situation, one can linearly minimize the magnitude of $v$, in order to maximize the information in the $z$ variables, in this case yielding $\left(v^{3}, z^{3}\right)=(-2.5,5,3,0)$. In our experience, however, the existence of a unique decomposition seems to be the rule rather than the exception.

In order to solve problem (QP) iteratively as constraints are generated, let $\bar{A}_{t}=\left(1, A_{t}\right)$, and after separating a new constraint, specified by $a_{C_{t}}$ and $\beta_{t}$, suppose we have solved the corresponding instance of 
(LP), determining $\mu_{t}$. Previous payment vector $p^{t-1}$ satisfies all known core constraints, except the new $C_{t}$ constraint, and may also not have high enough total payments to satisfy $p 1=\mu_{t}$. Given a decomposition $\left(v^{t-1}, z^{t-1}\right)$ of $p^{t-1}$, we may find the next decomposition $\left(v^{t}, z^{t}\right)$ by finding a feasible solution to the following linear system:

$$
\begin{aligned}
(d v, d z) \bar{A}_{t}^{T} \bar{A}_{t} & =\left(\delta_{0}, 0, \ldots 0, \delta_{t}\right) \\
d z & \geq-z^{t-1}
\end{aligned}
$$

and setting $\left(v^{t}, z^{t}\right)=\left(v^{t-1}, z^{t-1}, 0\right)+(d v, d z)$, where $\delta_{0}=\mu_{t}-\mu_{t-1}$, and $\delta_{t}=\beta_{t}-p^{t-1} a_{C_{t}}$. This result holds because of the interesting combinatorial form of $\bar{A}_{t}^{T} \bar{A}_{t}$, whose entries can be characterized as follows. First, let $R_{i}=W \backslash C_{i}$ represent the set that is responsible for overcoming a blocking offer made by $C_{i}$, and whose characteristic vector is given by $a_{C_{i}}$. Then defining $R_{0}=W$, and indexing the rows and columns of the symmetric matrix $\bar{A}_{t}^{T} \bar{A}_{t}$ starting at zero, it is easily shown that the row $r$, column $c$ entry of $\bar{A}_{t}^{T} \bar{A}_{t}$ is given by $\left|R_{r} \cap R_{c}\right|$. Then if we assess the impact of changing $(v, z)$ values by $(d v, d z)$ we see that the changes add up appropriately so that the $C_{t}$ th entry of $(d v, d z) \bar{A}_{t}^{T} \bar{A}_{t}$ gives the change in the total payments made by the group $R_{t}$, and thus the solution to this linear system provides an updated decomposition vector satisfying the KKT conditions. In practice, the constraint generation procedure typically only generates linearly independent core constraints, resulting in an invertible $\bar{A}_{t}^{T} \bar{A}_{t}$, in which case a unique solution to the linear system always exists. If it did happen that a linearly-dependent, loose constraint did arise (a solution will still exist if a lin. dep. constraint can remain tight), the addition of a single vector can cause at most one such dominated constraint, and we could, for example, remove constraints one at time until a solution exists, removing said dominated constraint from all remaining $A_{t+i}$ matrices.

\section{A.6 Practical Design Considerations for the U. K. Spectrum Auctions}

In the 10-40GHz auction, there were 27 lots auctioned, divided into 7 categories. Ofcom took an approach of first auctioning generic lots in a "principal stage," where each bid simply indicates quantities for each of the 7 categories. Each bidder then wins some quantity of generic (i.e., unspecified) licenses from each category, and we determined "base prices" for each of these bundles using the quadratic core-selection rule described here. This principal stage was then followed by an "assignment stage" in which a bidder who, for example, won 4 lots in Category A, could bid for which contiguous strip of 4 lots she actually wanted. Each category then had its own sealed-bid assignment stage auction with "top-up" bids, and the winners again paid the corresponding quadratic core prices, which were then listed as "additional prices" on top of their base price from the principal stage. As one would expect, these additional prices were small in comparison (sometimes 
zero) as there is little difference among the various specific assignments within a category.

A similar design is proposed for the upcoming $2.6 \mathrm{GHz}$ auctions (in both the U. K. and the Netherlands) but rather than fixed categories, the bidders specify instead the quantities of "paired" lots (separated by a fixed bandwidth) and how many singleton or "unpaired" lots they are interested in. Further, they can specify different preferences for groups of unpaired licenses that are split among two zones separated by a block of paired licenses. Again, a principal auction is used to determine who gets how many of each type of license, and is followed by an assignment stage to determine exactly which licenses are awarded to which bidders. Though the winner-determination problem for this auction is not as straight forward as formulation (WD), as long as we have some algorithm for solving winner determination problems the quadratic core-payment mechanism can be implemented. Indeed, even with different "blackboxes" for winner determination (for example, we used CPLEX's branching-based IP algorithm while dotEcon employed a dynamic programming technique) the uniqueness of the core selection strategy we present here guarantees that any technique will eventually produce the same outcome, making the validity of the process easy to verify.

The design choice to separate the auction into a principal generic stage and a specific assignment stage is helpful to guarantee computational quickness in the winner-determination problems we solve over the course of the algorithm, but is probably more valuable to reduce complexity for the bidders by not forcing them bid separately on nearly indistinguishable items. In the U. K.'s L-Band auction, this was not an issue, as the items being offered were 17 unique lots. Here the computational challenge was a separation requirement between high power and lower power usages, so that a bidder was required to reveal the intended usage of a package of lots within her bid, and the auction had to be sure that lots with dissimilar usages had at least two unused "guard bands" between them. Once a suitably tight IP formulation was determined, however, we were quickly able to solve winner determination problems for this 17 item auction with as many as 50,000 bids in just a few minutes, even in the worst case. 